\title{
LAS TENSIONES DE LA ESTRUCTURA DE LAS REVOLUCIONES CIENTIFICAS Y EL LEGADO DE THOMAS KUHN
}

\author{
THE TENSIONS OF THE STRUCTURE OF \\ SCIENTIFIC REVOLUTIONS AND THOMAS \\ KUHN'S LEGACY
}

\author{
LEONARDO DÍAZ* \\ Universidad del País Vasco
}

RESUMEN: Thomas Kuhn interpretó su obra como una filosofía de las prácticas científicas. Desde entonces, autores como Doppelt y Rouse proporcionaron una interpretación donde la perspectiva de Kuhn constituye una ruptura con la imagen de la ciencia como sistema de conocimientos. En este artículo mostraré que La estructura de las revoluciones científicas está constituida por la tensión existente entre una perspectiva de la ciencia como sistema de conocimientos y una perspectiva de la ciencia como sistema de prácticas, así como por un modelo dicotómico que le lleva a producir -a casi cincuenta años de su publicación- un legado ambiguo y paradójico.

Palabras Clave: Tensión, legado, sistema de conocimientos, sistema de prácticas, hermenéutica.

ABSTRACT: Thomas Kuhn interpreted his work as a philosophy of scientific practice. Since then, authors such as Doppelt and Rouse have offered an interpretation in which Kuhn's perspective represents a rupture with the image of science as a knowledge system. In this article I will show that The Structure of Scientific Revolutions is based upon a tension between a perspective of science as a knowledge system and a perspective of science as a system of practice, as well as by a dichotomous model that —almost 50 years after it was published — leads to an ambiguous and paradoxical legacy.

${ }^{*}$ E-mail del autor: rld_do@yahoo.com. 
KEYWORDS: Tension, legacy, knowledge system, system of practice, hermeneutics.

\section{Introducción}

Han transcurrido casi cincuenta años desde que el mundo académico fue impactado por la publicación de La estructura de las revoluciones científicas de Thomas Kuhn (1970). Desde entonces, los estudios críticos sobre esta obra no han cesado, tomando en los últimos diez años un giro conceptual significativo. Si durante las primeras décadas inmediatas a la publicación de La estructura los análisis giraron en torno a las ambigüedades de los conceptos explicativos básicos de Kuhn y en torno a las repercusiones epistemológicas de las nociones de ciencia normal e inconmensurabilidad, desde mediados de los años 90's emergieron estudios dedicados a analizar los vínculos de Kuhn con el empirismo lógico.

Los estudios de Bird (2000, 2004), Friedman (2003) y Gattei (2008) continuaron la línea de investigación iniciada en los años 90's por Reisch (1991), Axtell (1993), Earman (1993), Irzik y Grümberg (1995). Estos estudios mostraron cómo La estructura de las revoluciones científicas se enmarca en una línea de continuidad con postulados fundamentales del empirismo lógico, en vez de situarse como una ruptura radical de los mismos.

Paralelamente a estas investigaciones, otros análisis desde el campo de las ciencias cognitivas reforzaron la idea según la cual el programa de Kuhn constituye una ruptura con el empirismo lógico, por cuestionar el supuesto de la existencia de un conjunto de condiciones necesarias y suficientes para el aprendizaje del conocimiento científico y de un sistema explícito de reglas para su producción. En este contexto se inscriben los estudios de Andersen (2001), Nersessian (2003), Nickles (2003a) Andersen, Barker y Chen (2006).

Mientras tanto, desde los estudios culturales de la ciencia, Bernstein (1988), Rouse (1987), Rorty (1995) y Díaz (2008) situaron a Kuhn como un precedente significativo de un enfoque hermenéutico de la ciencia.

Como puede observarse, uno de los aspectos más notables de estas referencias es el hecho de que aglomeran un conjunto disímil de perspectivas, atri- 
buyendo a Kuhn su paternidad, o considerándolo como un importante precedente.

¿Cómo se explica esta variedad de enfoques, a casi cinco décadas de la publicación de su obra principal y cuál es su legado? Abordaré estos problemas a partir de una tesis sobre el modo en que dos perspectivas filosóficas configuran $\mathrm{La}$ estructura de las revoluciones científicas y que serán examinadas a continuación.

\section{La tensión entre una perspectiva de la ciencia como sistema de conocimientos y como sistema de prácticas}

En una evaluación de su propia obra, Kuhn (2000a) sostiene que la imagen de la ciencia representada por él reemplaza una perspectiva de la ciencia como cuerpo estático de conocimientos por una imagen dinámica de la ciencia, o una perspectiva de la ciencia como práctica.

Siguiendo esta idea, Doppelt (1978) cuestiona la postura de estudios críticos como los de Shapere $(1964,1971)$ o Sheffler (1967) donde la obra de Kuhn se analiza en términos meramente semánticos. Doppelt propone, por ejemplo, analizar el concepto kuhniano de inconmensurabilidad, entendiéndolo como un problema de comunicación existente entre las formas de vida de distintas comunidades científicas y no como un problema de incompatibilidad entre los significados de los marcos conceptuales de las mismas.

En la misma línea de pensamiento, Rouse (2003) sostiene que la filosofía de la ciencia ha sido considerada tradicionalmente en términos epistemológicos, esto es, como un sistema de conocimientos, y, por tanto, sus problemas giran en torno a la estructura, los procedimientos y la justificación del conocimiento científico. Por el contrario, propone Rouse, Kuhn articula una concepción de la ciencia como una actividad per se o una práctica.

Desde una perspectiva de la ciencia como sistema de conocimientos, la ciencia constituye un sistema de creencias justificadas, fundamentada en un conjunto explícito y universal de reglas. A partir de este sistema de conocimientos se genera la investigación científica misma, la cual está regida, en su estructura y aplicabilidad, por un método universal y homogéneo. Usualmente, un signo distin- 
tivo de esta perspectiva epistemológica es su preocupación por el problema de la estructura de las teorías científicas, los procesos de validación de las creencias, la naturaleza del método científico y una marcada despreocupación por los procesos reflexivos de adquisición del conocimiento ${ }^{1}$.

Estos procesos se perciben como propios del denominado contexto de descubrimiento, el proceso de generación del conocimiento científico. Desde un enfoque de la ciencia como sistema de conocimientos, el contexto de descubrimiento es de naturaleza psicológica, porque se relaciona con problemas como el de la creatividad o el papel que juega la imaginación en los procesos de construcción cognoscitiva. Desde este punto de vista resulta, por tanto, carente de interés para la filosofía de la ciencia, porque se entiende que no puede ser reconstruido desde el punto de vista racional.

A diferencia del contexto de descubrimiento, el contexto de justificación se relaciona con el proceso de validación de las hipótesis científicas ${ }^{2}$. Desde la perspectiva de la ciencia como sistema de conocimientos, este es el verdadero objeto de la reflexión epistemológica. La filosofía de la ciencia es fundamentalmente una reflexión sobre los métodos de la ciencia, no sobre el origen de las ideas científicas.

En contraste, una perspectiva de la ciencia como sistema de prácticas concibe la ciencia a partir de las actividades producidas por las comunidades epistémicas. El conocimiento científico es el producto de estas actividades que implican el uso de instrumentos y tecnologías, así como de procesos no reflexivos o conocimientos tácitos. La preocupación gira en torno a la dinámica de las teorías científicas y se entiende que no existe una distinción tajante entre el contexto de descubrimiento y el contexto de justificación. Por consiguiente, el proceso de adquisición del conocimiento es de sumo interés desde el punto de vista de la reflexión epistemológica.

Al mismo tiempo, desde esta perspectiva, las investigaciones científicas se ins-

\footnotetext{
${ }^{1}$ Para esta perspectiva, véase Ayer (ed.) (1965), Carnap (2002).

${ }^{2}$ La distinción entre contexto de descubrimiento y contexto de justificación es atribuida comúnmente a Hans Reichenbach. No obstante, Losee (2001) señala que la distinción se debe originariamente a John Herschel, en 1830, reformulada por Reichenbach en 1938.
} 
criben en el contexto de tradiciones de investigación que están caracterizadas por su historicidad. De ahí que la empresa de la ciencia se defina como dinámica e históricamente condicionada ${ }^{3}$.

Sostendré en este artículo que, a diferencia de la autoimagen de Kuhn y de las interpretaciones de Doppelt y Rouse, La estructura de las revoluciones científicas no constituye una ruptura radical con la imagen de la ciencia como sistema de conocimientos.

Por el contrario, sostendré que La estructura de las revoluciones cientificas está constituida por la tensión existente entre una perspectiva de la ciencia como sistema de conocimientos similar a la del empirismo lógico ${ }^{4}$ y una perspectiva de la ciencia como sistema de prácticas. Empleo el término de tensión como una metáfora que designa el estado de un sistema conceptual conformado por perspectivas opuestas que coaccionan el sistema, generando ambigüedades y contrastes en los términos explicativos básicos, así como en las explicaciones derivadas del mismo.

La existencia de las dos perspectivas señaladas dentro de la epistemología kuhniana se muestra en el contraste existente por un lado, entre el énfasis de Kuhn por explicar el proceso de aprendizaje y funcionamiento de la actividad científica, minimizando la función de las teorías y los procesos reflexivos, y por el otro lado, su insistencia en el papel que desempeñan las teorías como fundamento para la percepción y el ordenamiento de los datos científicos, la configuración de las especialidades y las revoluciones científicas.

Kuhn insiste en el carácter determinador de las teorías como base para la percepción, pues las mismas condicionan el modo de ver de las comunidades científicas. Al mismo tiempo, las teorías determinan el desarrollo de las prácticas científicas futuras. Sin embargo, al contrario de lo que ocurre en la imagen de la ciencia como sistema de conocimientos, en la perspectiva de Kuhn las teorías no se aprenden tanto a través de un sistema de aprendizaje de axiomas y reglas de deducibilidad, sino a través del aprendizaje de conceptos contextualizados en prácticas,

${ }^{3}$ Para una perspectiva de la ciencia como sistema de prácticas, véase: Kuhn (2000a), Doppelt (1978), Hacking (1996), Pickering (1995) y Rouse (1996, 2003).

${ }^{4}$ Sobre los vínculos de Kuhn con el empirismo lógico, cf. Reisch (1991), Irzik y Grümberg (1995), Axtell (1993), Earman (1993), Friedman (2002), Bird (2000, 2004), Gattei (2008). 
del desarrollo de un conocimiento tácito, que por definición, no es teórico. Por un lado, las teorías son determinantes de las prácticas, por otra parte, son su resultado. Por una parte, las teorías ocupan un lugar determinante en el desarrollo de la ciencia, por el otro, el papel de las teorías se minimiza, privilegiando una dimensión cognoscitiva tácita 5 .

Kuhn (1970) introduce la noción de paradigma en la postdata a La estructura de las revoluciones científicas. El ejemplar es un modelo de resolución de problemas. De acuerdo con esta perspectiva, el proceso mediante el cual se aprenden los ejemplares no es teórico, no forma parte de un conocimiento explícito, más bien se asemeja a procesos no intelectivos como los descritos por Wittgenstein (1988) y Polanyi (1964).

El ejemplar funciona como un modelo a partir del cual el estudiante aprende a establecer relaciones de semejanza y disimilitud. Esta perspectiva del aprendizaje conceptual de la ciencia no requiere de la existencia de un sistema de reglas. Según Kuhn $(1970,1977)$, el aprendizaje se produce por una exposición a ejemplos con los cuales se adquiere inconscientemente la habilidad de relacionar y diferenciar objetos. El proceso es explicable desde el modelo conexionista de las ciencias cognitivas. Parafraseando el ejemplo de Bird (2000), los integrantes de una comunidad se entrenan a partir de una serie de estímulos que activa la red de conexiones cerebrales como las entradas (inputs) de un ordenador. En una determinada entrada, se activan las respuestas vinculadas con el estímulo, de modo que si el mismo consiste en una estimulación sensorial, por ejemplo, un estímulo visual proveniente de una película, la respuesta provocada por la red puede ser el nombre de un lugar recordado, del autor del film, etc. Dependiendo de las respuestas, el cerebro modifica por azar las conexiones entre las entradas (inputs) y salidas (outputs). Si las mismas no se relacionan -el estímulo visual ha sido atribuido errónea-

\footnotetext{
${ }^{5}$ Para este contraste véase Kuhn (1970, capítulos I, V, X y postdata).

${ }^{6}$ Kuhn no define claramente la noción de teoría. Emplea el término tanto para referirse a un cuerpo de creencias (1970, p. 2), así como para referirse a un sistema de compromisos conceptuales, problemas y normas (pp. 6-7), a un sistema explicativo de hechos (pp. 17-18), o para una cosmovisión (Ver la posdata). Lo más destacable es que a pesar de estas diferencias, para Kuhn, la noción de teoría implica siempre un proceso reflexivo que se contrapone a l conocimiento tácito implícito en la noción de ejemplar. En torno a este concepto se fundamenta su filosofía de las prácticas científicas.
} 
mente a un lugar, o a un autor equivocado- el cerebro debilita la conexión, o la refuerza en caso de que sí exista la relación ${ }^{7}$.

Este proceso de reforzamiento neuronal posibilita el desarrollo de la capacidad cognitiva para establecer correctamente relaciones de semejanza y disimilitud.

Del mismo modo, los integrantes de una comunidad científica son expuestos a ejemplos de resolución de problemas que estimulan el establecimiento de relaciones de semejanza y disimilitud reforzables neuronalmente por el proceso anteriormente descrito. Nótese que se trata de un proceso inconsciente para los agentes implicados. No hay reglas ni teorías aprendidas, como tampoco procesos de inferencia.

Este enfoque que minimiza el papel de la teoría y de los procesos reflexivos pugna en la obra de Kuhn con una perspectiva que sobrestima el papel que juegan las teorías en los procesos epistémicos ${ }^{8}$. Kuhn es compromisario de una perspectiva de la ciencia como sistema de conocimientos similar a la de autores como Rudolf Carnap y Norwood Hanson. Carnap (2002) defendió la preeminencia de los marcos lingüísticos en el modo de describir el mundo, así como en los valores de verdad asignados a dichas descripciones. Por su parte, Hanson (1977) defendió la tesis de la carga teórica de las observaciones. Según esta tesis, las observaciones están indisolublemente vinculadas a las teorías y creencias de los observadores, de tal modo que no existen datos puros. Toda observación es un acto interpretativo.

Kuhn asumió ambos supuestos. Asignó a las teorías y marcos conceptuales un papel fundamental en la constitución de las disciplinas científicas y en la constitución del mundo. Asumiendo la concepción ontológica de Kant, defendió la existencia de una realidad impensable e incognoscible contrapuesta a una realidad fenoménica que se constituye a partir de las creencias y teorías de las comunidades científicas ${ }^{9}$. Por esta razón, en la epistemología kuhniana, cuando cam-

\footnotetext{
${ }^{7}$ Véase Bird (2000, p. 74).

${ }^{8}$ Compárese al respecto Kuhn (1970, caps. II, V, X y posdata).

${ }^{9} \mathrm{La}$ ambigüedad de Kuhn en su noción de mundo - entendido como realidad nouménica, intratable, incognoscible e impensable, y por otra parte, entendido como una realidad fenoménica o realidad conceptualizada - posibilitó diversas interpretaciones que terminaron apadrinando
} 
bian las creencias de los agentes epistémicos, se modifican también sus percepciones y el mundo mismo de la comunidad científica.

De este modo, dentro de una filosofía de las prácticas científicas las teorías y las creencias desempeñan la función de fundamento. Esta tensión genera una serie de ambigüedades para la terminología kuhniana. Por un lado, introduce la noción de paradigma — como ejemplar- porque según sus propias palabras, quiere distanciarse de la noción tradicional de teoría ${ }^{10}$. En este sentido, la noción de paradigma entendida como ejemplo de resolución de problemas se constituye en la fuente del consenso de las comunidades epistémicas y en el fundamento de una filosofía de las prácticas científicas. Pero este sentido contrasta con el concepto de paradigma como teoría predominante, que se deriva de los compromisos de Kuhn con una pespectiva de la ciencia como sistema de conocimientos.

Por el mismo motivo, otra de las nociones básicas de la teoría kuhniana de la ciencia, el concepto de inconmensurabilidad, remite tanto a problemas de incompatibilidad a nivel de las prácticas de las comunidades epistémicas, así como a problemas de incompatibilidad semántica ${ }^{11}$.

programas rivales. Un programa se focalizó en la noción de mundo en su sentido fenoménico, defendiendo con ello el carácter construido de los datos científicos y la inexistencia de una realidad objetiva. Esta fue la orientación que tomaron los programas escépticos y relativistas, como el programa fuerte de la sociología del conocimiento científico. Véase Latour y Woolgar (1995).

Por otro lado, otro enfoque recuperó la noción de realidad nouménica o no conceptualizada, interpretando a Kuhn en los términos de un tipo de realismo interno al estilo de Hilary Putnam. Véase Pérez Ransanz y Alvarez (2004) y Gattei (2008).

Así, la tensión existente en la ontología kuhniana entre sus dos nociones de mundo produce una perspectiva que titubea entre el carácter no construido de la realidad y el carácter construido de la misma. Con ello, se genera una tensión en el modelo explicativo, entre la ciencia como generadora de hallazgos y la ciencia como empresa creadora. Al respecto, véase Kuhn (1970, 2000b).

${ }^{10}$ Cf. La postdata.

${ }^{11}$ Kuhn utiliza doce veces el concepto de inconmensurabilidad en La estructura de las revoluciones cientificas, sin definirlo explícitamente. (Kuhn, 1970, pp. 4, 103, 112, 148, 149, 150, 157, $165,175,198,200)$. Esto contribuyó a muchos de los malentendidos del vocablo. Posteriormente, Kuhn (2000c) señala que extrajo el vocablo de las matemáticas, aludiendo con él a la inexistencia de un lenguaje neutral donde puedan traducirse dos teorías, como conjunto de enunciados, sin que haya pérdida semántica en la traducción. Sin embargo, el problema es más complejo, porque Kuhn empleó distintos sentidos del término inconmensurabilidad. Véase al respecto, Sankey (1993,1994). 
Ante la ausencia de un cuerpo de creencias o de teorías, Kuhn sostiene que no hay consenso sobre los criterios metodológicos ni sobre el modo de interpretar los datos ${ }^{12}$. Para una filosofía de las prácticas científicas, el problema del consenso epistémico no adquiere la relevancia que tiene para una perspectiva de la ciencia como sistema de conocimientos, ni se explica en función de la teoría que comparte una determinada comunidad científica. Como he señalado, en la imagen de Kuhn éstas parecen ser las determinantes para explicar el problema del consenso.

Sin embargo, en el capítulo V de La estructura y posteriormente, en La posda$t a$, Kuhn fundamenta el problema del consenso en las prácticas de las comunidades científicas. Esto hace innecesario la existencia de un acuerdo a nivel de las teorías:

«... a theory of matter is not the sort of topic on which the members of even a single community must necessarily agree. The need for agreement depends o what it is the community does». (1970, p. 180) ${ }^{13}$.

La importancia asignada por Kuhn al problema del consenso, le lleva a sustituir el concepto de sistema de reglas característico de las concepciones de la ciencia como sistema de conocimientos, por la noción de paradigma como teoría coercitiva. Vinculado a esta noción, construye un modelo dicotómico que describe la ciencia constituida por períodos de investigación acrítica regidos por una teoría, interrumpida por fases de ruptura innovadora a los que denomina ciencia extraordinaria ${ }^{14}$. Esto nos lleva a la segunda tensión que examinaré en este artículo.

\footnotetext{
${ }^{12}$ Kuhn (1970, cap. II).

${ }^{13}$ Kuhn emplea en esta cita su concepto más vago de teoría —algo más parecido a una cosmovisión - pero esto no altera mi tesis, puesto que su idea básica es que las comunidades científicas pueden funcionar efectivamente a partir de ejemplares o modelos de resolución de problemas - un tipo de conocimiento no teórico — sin la necesidad de un acuerdo a nivel de cosmovisiones y de racionalizaciones. Cf. Kuhn (1970, cap. V).

${ }^{14}$ Véase al respecto, Kuhn (1970, caps. III, IV y IX).
} 


\section{La tensión entre la ciencia como empresa conservadora y como empresa innovadora}

En La estructura de las revoluciones cientificas, Kuhn defiende la existencia de un período denominado ciencia normal caracterizado por la ausencia de innovación. Describe esta fase como un periodo de resolución de rompecabezas (problem solving). La metáfora debe entenderse en un sentido fuerte que implica:

a) La existencia de una fase de soluciones anticipadas a los problemas formulados que sólo la falta de habilidad de los integrantes de la comunidad científica impide alcanzar.

b) Una creencia incuestionable en los fundamentos de la tradición que sirve de base para la investigación.

c) La irrelevancia de la utilidad.

d) La ausencia de innovación.

Kuhn (1970) afirma que existen tres núcleos básicos de problemas que resumen la investigación científica normal: La determinación de los hechos significativos, la adecuación de los hechos a la teoría predominante y el proceso de refinamiento en la articulación de la misma. La característica común a estos tres núcleos de problemas es la acriticidad. Abordar estas cuestiones no exige de quienes se dedican a ellos cuestionar los supuestos básicos de la tradición que sustenta la investigación científica normal. Es esta acriticidad la que permite el desarrollo de la ciencia normal ante la ausencia de un sistema de reglas:

«Normal science can proceed without rules only so long as the relevant scientific community accepts without question the particular problem-solutions already achieved». (Kuhn, 1970, p. 47).

Por tanto, la dinámica de la ciencia tiende a la preservación de los contenidos cognitivos. Esta dinámica se interrumpe cuando surge el período de ciencia extraordinaria a raíz de una revolución científica:

«...the preceding discussion has indicated that scientific revolutions are here taken to be those non-cumulative developmental episodes in which an 
older paradigm is replaced in whole or in part by an incompatible new one». (Kuhn, 1970, p. 92).

Por tanto, el modelo explicativo de Kuhn crea una tensión entre un período caracterizado por la acriticidad y la carencia de innovación por un lado, y por el otro, un período caracterizado por la criticidad y la innovación. Uno de los problemas básicos de este modelo consiste en explicar los períodos de discontinuidad conceptual a partir de los períodos de continuidad conceptual y dogmatismo. Kuhn no soluciona satisfactoriamente este problema y permanece comprometido con este modelo cuando, varias décadas después de los debates del Bedford College ${ }^{15}$ en torno a la naturaleza de la ciencia normal, protagoniza un debate con el filósofo canadiense Charles Taylor.

El 11 de febrero de 1989, Kuhn fue invitado a participar en un panel con Taylor, sobre la distinción entre las ciencias naturales y las ciencias humanas. Taylor (1985) había sostenido cuatro años antes la clásica distinción de Dilthey (1986) entre ciencias naturales y ciencias del espíritu. Según esta clasificación, las primeras son empresas explicativas carentes de toda determinación histórica y cultural, mientras las ciencias humanas son empresas comprensivas o hermenéuticas con sistemas de significados condicionados histórica y culturalmente. Para Taylor, los objetos de las ciencias naturales carecen de significado y si lo tienen, es el mismo para todos.

Kuhn (2000d) cuestiona la demarcación de Taylor sosteniendo que los objetos de las ciencias naturales sí tienen significado y que están determinados histórica y culturalmente, debido a que las entidades del mundo se estructuran a partir de un léxico dado por la cultura.

El problema que subyace al debate Kuhn-Taylor es el de la naturaleza de los datos científicos. Taylor entiende que las ciencias naturales poseen datos puros, a diferencia de las ciencias humanas cuyos datos están condicionados por las interpretaciones del investigador.

\footnotetext{
${ }^{15}$ Los debates celebrados en el Bedford College, en Londres, fueron recopilados por Lakatos y Musgrave (eds.) (1970).
} 
En La estructura de las revoluciones cientificas, Kuhn sostuvo tajantemente la inexistencia de datos puros y fue consistente con esta postura hasta el final de su trayectoria intelectual ${ }^{16}$.

En su debate con Taylor y a partir de esta postura, Kuhn pone en entredicho la distinción entre ciencias naturales y humanas, pero da un giro al problema sosteniendo la paradoja de que las ciencias naturales, si bien tienen una base hermenéutica, carecen de prácticas hermenéuticas. Afirma que a diferencia de lo que acontece en las ciencias humanas, el marco conceptual proporcionado por la tradición no proporciona un fundamento para la interpretación, sino para la resolución de problemas. Lo que sale a relucir aquí son los compromisos de Kuhn con el modelo dicotómico ciencia normal-ciencia revolucionaria. Si la tradición realizara en las ciencias naturales la misma función de base para múltiples interpretaciones, entonces no habría lugar para un período como el de la ciencia normal y por tanto, para la preservación de la investigación.

En la perspectiva de Kuhn, la práctica de las comunidades científicas está dirigida hacia la autopreservación de la tradición de investigación científica que ha contribuido a conformar la disciplina. Este ejercicio impide la innovación. Si las prácticas científicas no estuvieran dirigidas hacia la perpetuación de los fundamentos de la tradición y la disciplina, entonces la investigación científica estaría dirigida hacia la constante reformulación de la misma. Para Kuhn, esta constante reformulación impediría su progreso.

Desde esta perspectiva, la preservación es un valor más estimado que la innovación. A diferencia del cultivador de las humanidades, cuyo prestigio está más relacionado con su capacidad de reinventar un estilo, una escuela o un sistema de pensamiento, el científico normal de Kuhn es valorado en la medida en que muestra sus habilidades en la resolución de los problemas que le ha proporcionado su tradición de investigación.

Por tanto, desde la perspectiva de Kuhn, la innovación caracteriza a las ciencias humanas, la preservación a las ciencias naturales. Estas, a su vez, experimentan fases de continuidad y carencia de innovación interrumpidas por fases de creatividad.

${ }^{16}$ Véase al respecto, Kuhn (1970, cap. X, 2000b, 2000d). 
Aferrándose a este modelo, Kuhn replantea la dicotomía entre ciencias naturales y ciencias humanas sobre la base de la dualidad preservación-innovación y, de este modo, refuerza la demarcación cuestionada en su debate con Taylor. Dicho modelo no dejó de tener consecuencias para su legado.

\section{El legado de Kuhn}

No es de extrañar que las tensiones explicadas anteriormente generaran un legado ambiguo y paradójico. Por un lado, los compromisos de Kuhn con la perspectiva de la ciencia como sistema de conocimientos le hicieron compromisario de un legado empirista similar al de Carnap (2002), quien enfatiza la función de los marcos conceptuales como determinantes de la actividad científica.

Por otro lado, partiendo del Wittgenstein de las Investigaciones filosóficas y de Polanyi, desarrolló una filosofía de las prácticas científicas que minimiza la función de los marcos conceptuales y describe los procesos de aprendizaje conceptual en términos no intelectivos. Recuperando para la filosofía de la ciencia la noción de conocimiento tácito, desarrolló una perspectiva de la cognición que toma distancia de las perspectivas intelectualistas de la ciencia.

Debatiéndose entre fuentes que priorizaban el papel de las teorías y otras que priorizaban el papel de las prácticas, Kuhn desarrolló su propio modelo explicativo integrando ambas perspectivas incoherentemente.

La tensión entre la perspectiva de la ciencia como sistema de conocimientos y la perspectiva de la ciencia como sistema de prácticas generó la ambigüedad de Kuhn en el uso de sus conceptos claves. La noción de paradigma, empleada en el sentido de cosmovisión y de teoría, permitió la invocación de Kuhn para referirse a todo tipo de marco conceptual o enfoque, a pesar de su intención de abandonar el sentido tradicional de teoría.

Al mismo tiempo, empleando el concepto de paradigma como ejemplar, introdujo una noción que explicaba el aprendizaje conceptual en términos no algorítmicos. Con ello, construyó un legado para las investigaciones de línea cognitiva como las de Andersen, Barker y Chen (2006), o Nickles (2003a), entre otros. 
A partir de la noción de paradigma como ejemplar articuló una descripción de la ciencia en función de las prácticas de las comunidades científicas, creando un legado para fundamentadores de una filosofia de las prácticas científicas como Pickering (1995), Hacking (1996), o Rouse (1996, 2002).

El concepto de inconmensurabilidad experimentó un contraste similar. Por un lado, acorde con la noción más teorética de paradigma, su empleo estimuló interpretaciones que enfatizaban la incompatibilidad semántica de Kuhn, en la línea de autores como Shapere (1964, 1971), Scheffler (1967), o Sankey (1993, 1998, 1999).

Por otra parte, la noción no teorética de inconmensurabilidad, posibilitó su interpretación en términos de una filosofía de las prácticas científicas como la de Doppelt (1978).

La segunda tensión aludida en este artículo también generó ambigüedades para el legado de Kuhn.

La perspectiva de Kuhn sobre la naturaleza de la ciencia generó una tradición crítica que interpretó su legado como propagador de formas de vida violentas, de corrientes antihumanitarias y autoritarias ${ }^{17}$ o, en los términos de Fuller $(2000,2004)$, como el promotor de una ideología conservadora de la Guerra Fría destinada a defender a la ciencia de la regulación ciudadana, mientras protegía del cuestionamiento a los responsables de las políticas científicas del sistema norteamericano.

Al mismo tiempo, la tesis kuhniana sobre las revoluciones científicas, el carácter discontinuo de la ciencia y la sociología de los grupos científicos, convirtieron a Kuhn en un héroe intelectual de sectores liberales que, como los defensores del programa fuerte de la sociología del conocimiento científico, percibieron su obra como una desmitificación de la actividad científica y con ello, del último reducto de experiencia desvinculado de la influencia de los intereses ideológicos.

\footnotetext{
${ }^{17}$ Para el primer caso, véase Lakatos (1970). Como promotor de tendencias antihumanitarias, véase Feyerabend (1970). Como promotor del autoritarismo, véase Gattei (2008).
} 
La obra de Kuhn estuvo vinculada con estos enfoques y, a la vez, a distancia de ellos. Vinculada, en la medida en que estas interpretaciones surgieron de la lectura basada en las ambigüedades de los textos de Kuhn. A distancia, en la medida en que eran interpretaciones sesgadas por haberse centrado en uno de los polos de las tensiones filosóficas que las producían.

Los planteamientos de Kuhn sobre el carácter histórico y cultural de la ciencia, abrieron también un legado para los estudios culturales de la ciencia. Desde su perspectiva, las mismas categorías que han sido empleadas para comprender fenómenos considerados tradicionalmente culturales pueden ser empleadas para el análisis de la ciencia, pues ella no es una empresa menos cultural que el arte o las humanidades.

La introducción de su perspectiva de la ciencia como sistema de prácticas basadas en tradiciones particulares de investigación y enraizadas en un contexto cultural determinado constituye de hecho, el principal legado de Kuhn para los estudios culturales de la ciencia.

Pero al mismo tiempo, Kuhn mantuvo posturas que lo confrontan significativamente con una perspectiva cultural de la ciencia haciéndolo incoherente con su propio programa de investigación.

Desde la perspectiva de los estudios culturales de la ciencia, la actividad científica está íntimamente vinculada a las tecnologías. La obra de Kuhn desvincula ambas actividades. Si bien afirma que la tecnología ha desempeñado una función crucial en el surgimiento de determinadas ciencias ${ }^{18}$, omite la mayor parte del tiempo las referencias a las relaciones entre ciencia y tecnología. Algunos fragmentos de La estructura orientan sobre cuál es la perspectiva de Kuhn sobre esta relación, al afirmar que la autonomía de las comunidades científicas con respecto a la intervención ciudadana, constituye una parte de las reglas no escritas del juego científico. Este es el argumento subyacente a la distinción kuhniana entre ciencia y tecnología. Kuhn distingue las ciencias de las disciplinas tecnológicas, porque las primeras son actividades autorreguladas, mientras las segundas se desarrollan presionadas por la sociedad civil:

\footnotetext{
${ }^{18}$ Cf. Kuhn (1970a) cap. II.
} 
«(...) the insulation of the scientific community from society permits the individual scientist to concentrate his attention upon problems that he has good reason to believe he will be able to solve. Unlike the engineer, and many doctors, and most theologians, the scientist need not choose problems because they urgently need solution and without regard for the tools available to solve them (...)». (1970, p. 164).

La demarcación kuhniana entre ciencia y tecnología se dirige a la preservación de la ciencia con respecto a la regulación pública y a la intromisión ciudadana que es común a la tecnología. Kuhn afirma que el aislamiento de la comunidad científica con respecto al resto de la sociedad posibilita el progreso científico. Este argumento no se sostiene si observamos que las disciplinas tecnológicas igualmente progresan en la resolución de problemas, teniendo una relación directa con la sociedad civil. Además, la historia de la ciencia muestra suficientes ejemplos de cómo la influencia de la sociedad civil contribuye al progreso de las disciplinas científicas ${ }^{19}$.

La perspectiva de Kuhn sobre las diferencias entre ciencia y tecnología ha tenido importantes repercusiones para la interpretación de su legado. Al igual que Fuller $(2000,2004)$, Rouse $(1991,1996)$ interpreta a Kuhn como un conservador, situando su perspectiva en el contexto del debate Bernal-Polanyi. La posición de J. D. Bernal asume como propio de la investigación científica una finalidad social, vinculándola a la sociedad civil y defendiendo la necesidad de que sea regulada por la misma. Por el contrario, la perspectiva de Polanyi sobre el carácter tácito del conocimiento hace de los practicantes de una determinada disciplina científica los únicos responsables de su producción y su evaluación. Por tanto, desde su perspectiva, cualquier orientación de la ciencia, promovida desde el exterior de las comunidades científicas, socava la naturaleza misma de la empresa científica.

Por los mismos motivos que Polanyi, Kuhn proclamó la autonomía absoluta de las comunidades científicas con respecto al resto de la sociedad, defendiendo una postura que lo opone a la perspectiva de los estudios culturales de la ciencia.

${ }^{19}$ Véase Goldstein (1991), Benoit (1991), Stengers (1991), Authier (1991). 
Así, y a modo de conclusión, la obra de Kuhn proporcionó un legado paradójico. Si su perspectiva de la ciencia como sistema de prácticas, su énfasis en la función cognoscitiva de las tradiciones locales de investigación y su mirada cognitiva de los procesos epistémicos constituyeron un legado de importancia indudable para las investigaciones cognitivas, la filosofía de las prácticas científicas y los estudios culturales de la ciencia, sus compromisos empiristas, que le llevaron a su concepto de paradigma como teoría coercitiva, su descripción de un período de acriticidad denominado ciencia normal y su tesis sobre el aislamiento de los colectivos científicos del resto de la sociedad, constituyeron perspectivas contradictorias a enfoques que contribuyó a estimular.

Como puede apreciarse, las tensiones de La estructura produjeron para la filosofía postkuhniana de la ciencia un legado tan paradójico y ambiguo como lo fue la misma obra de Kuhn.

\section{Bibliografía}

Andersen, H. (2001). On Kuhn, Wadsworth, Belmont CA.

ANDERSEN, H., BARKeR, P. y CHEN, X. (2006). The cognitive structure of scientific revolutions, Cambridge University Press, Cambridge.

Authier, M. (1991). «¿Ciencia pura, ciencia aplicada, ciencia militar?» En M. Serres (ed.) (1991), pp.119-149.

AxTeLL, G. (1993). «In the tracks of the historicist movement. Re-assessing the CarnapKuhn connection». Studies in History and Philosophy of Science, 24: 119-146.

AYer. A., ed. (1965). El positivismo lógico, FCE, México.

BENoIT, P. (1991). «¿Una aritmética o dos?: Cálculo, álgebra y mercancía». En M. Serres (ed.) (1991), pp. 225-253.

Bernstein, R. (1988). Beyond objectivism and relativism: Science, hermeneutics, and praxis, University of Pennsylvania Press, Philadelphia.

BiRD, A. (2000). Thomas Kuhn, Princeton University Press, Princeton. (Trad. cast. 2002, Thomas Kuhn, Ed. Tecnos, Madrid).

- (2004). «Kuhn, naturalism, and the positivist legacy». Studies in History and Philosophy of Science, 35: 337-356.

CARnAP, R. (2002). The logical syntax of language, Open Court, Chicago. 
Conant, J. y Haugeland, J., eds. (2000). The road since structure, Chicago: The University of Chicago Press. (Trad. cast. 2002, El camino desde la estructura, Ed. Paidós, Barcelona).

DiAZ, L. (2008). «Paradigmas-matrices y paradigmas-ejemplares: Entre una hermenéutica de la ciencia y una filosofía de las prácticas científicas». En G.Vatttimo, T.Oñate, A.Nuñez y F. Arenas (eds.) (2008), 117-143.

DiLTheY, W. (1986). Introducción a las ciencias del espíritu, Alianza Editorial, Madrid.

Doppelt, G. (1978). «Kuhn's epistemological relativism: A interpretation and defense. Inquiry, vol. 21: 33-86.

EARman, J. (1993). «Carnap, Kuhn, and the philosophy of scientific methodology». En P. Horwich (ed.) (1993), 9-36.

Feyerabend, P. (1970). «Consolations for the specialist». En I. Lakatos y A. Musgrave (eds.) (1970), op. cit., pp. 197-230.

Friedman, M.(2003). «Kuhn and logical empiricism». En T. Nickles (2003b) (ed.), pp. 19-44.

Fuller, S. (2000). Thomas Kuhn: A philosophical history for our time, The University of Chicago Press, Chicago.

- (2004). Kuhn vs. Popper: The struggle for the soul of science, Columbia University Press, New York.

GatTeI, S. (2008). Thomas Kuhn's linguistic turn and the legacy of logical empiricism: Incommensurability, rationality and the search for truth, Ashgate Publishing Limited, Aldershot, Hampshire.

Goldstein, C. (1991). «El uno es el otro: una historia del círculo». En M. Serres (ed) (1991), pp. 151-173.

Hacking, I. (1996). Representar e intervenir, Ed. Paidós, México.

Hanson, N. (1977). Patrones de descubrimiento, Alianza Editorial, Madrid.

HorwiCH, P., ed. (1993). World changes: Thomas Kuhn and the nature of science, The MIT PRESS, Cambridge.

IrZIK, G. y GrumberG, T. (1995). "Carnap and Kuhn: Arch enemies or closes allies?» The British Journal for the Philosophy of Science, 46: 285-307.

Kunn, T. (1970). The Structure of scientific revolutions, The University of Chicago Press, Chicago (Trad. cast. 2004, La estructura de las revoluciones cientificas, FCE, México).

— (1977). «Second thoughts on paradigms». En T. Kuhn (ed.), The essential tension: 
Selected studies in scientific tradition and change, The University of Chicago Press, Chicago, pp. 293-319. (Trad. cast. 1982, "Algo más sobre los paradigmas», en La tensión esencial, FCE, México, pp. 317-343).

- (2000a). «The trouble with the historical philosophy of science». En J. Connant and J. Haugeland (eds.) (2000), pp. 105-120.

- (2000b). «The road since structure». En J. Conant y J. Haugeland (eds.), (2000), pp. 90-104.

- (2000c). «Commensurability, comparability and communicability». En J. Conant y J. Haugeland (eds.) (2000), pp. 33-57.

- (2000d). «The natural and the human sciences». En J. Conant y J. Haugeland (eds.) (2000), pp. 216-223.

Lakatos, I. y Musgrave, A., eds. (1970). Criticism and the growth of knowledge, Cambridge University Press, Cambridge. (Trad. cast. 1975, La critica y el desarrollo del conocimiento, Ediciones Grijalbo, Barcelona, pp. 203-343).

- (1970). «Falsification and the methodology of scientific research programmes». En I. Lakatos y A. Musgrave (eds.) (1970), pp. 91-196. (Trad. cast. 1975. La critica y el desarrollo del conocimiento, Ediciones Grijalbo, Barcelona, pp. 203-343).

Latour, B. y WoOlgar, S. (1995). La vida en el laboratorio, Alianza Universidad, Madrid.

LOSEE, J. (2001). A historical introduction to the philosophy of science, Oxford University Press, New York.

Nerssesian, N. (2003). «Kuhn, conceptual change, and cognitive science». En T. Nickles (ed.) (2003b), pp. 178-211.

NiCKLES, T. (2003a). «Normal science: From logic to case-based and model-based reasoning». En T. Nickles (ed.) (2003b), pp. 142-177.

- (ed.) (2003b). Thomas Kuhn, Cambridge University Press, Cambridge.

Perez Ransanz, A. R. y Alvarez, J. F. (2004). «De Kant a Kuhn, acotando por Putnam». Endoxa, Series Filosóficas, 18: 495-517.

Pickering, A. (1995). The mangle of practice: Time, agency, and science, The University of Chicago Press, Chicago.

Polanyi, M. (1964). Personal Knowledge: Towards a post-critical philosophy, Harper and Row, New York.

ReISCH, G. (1991). «Did Kuhn Kill Logical Empiricism?. Philosophy of Science, 58: 264277. 
RorTy, R. (1995). La filosofía y el espejo de la naturaleza, Ed. Cátedra, Madrid.

Rouse, J. (1987). Knowledge and power: Toward a political philosophy of science. Cornell University Press, Ithaca.

- (1996). Engaging science: How to understand its practices philosophically, Cornell University Press, Ithaca.

- (2002). How scientific matter: Reclaiming philosophical naturalism, The University of Chicago Press, Chicago.

— (2003). «Kuhn's Philosophy of Scientific Practice». En T. Nickles (ed.) (2003b), op. cit., pp. 101-121.

SANKEY, H. (1993). «Kuhn's changing concept of incommensurability». British Journal for the Philosophy of Science, 44: 775-791.

- (1998). "Taxonomic incommensurability». International Studies in the Philosophy of Science, 12: 7-16.

- (1994). The incommensurability thesis, Avebury, Aldershot.

— (1999). «Incommensurability -an overview». Divinitatio, 10: 1-13.

Scheffler, I. (1967). Science and subjectivity, The Bobbs-Merrill Company, Inc, New York.

Serres, M., ed. (1991). Historia de las ciencias, Ed. Cátedra, Madrid.

Shapere, D. (1964). "The structure of scientific revolutions». Philosophical Review, 73: 383-394.

- (1971). "The paradigm concept». Science, 172: 703-709.

STENGERS, I. (1991). «Los episodios galileanos». En M. Serres (ed.) (1991), pp. 255-285.

TAYLOR, C. (1985). "Interpretation and the sciences of man». En C. Taylor, Philosophy and the human sciences (vol. 2), Cambridge University Press, Cambridge, pp. 15-57.

Vatтimo, G., Oñate, T., Nuñez, A. y Arenas, F., eds. (2008). El mito del uno: Horizontes de latinidad: Hermenéutica entre civilizaciones, Ed. Dykinson, Madrid.

WitTGenstein, L. (1988). Investigaciones filosóficas, Ed. Crítica, Barcelona.

Recibido: 28/04/2010

Aceptado: 8/06/2010 